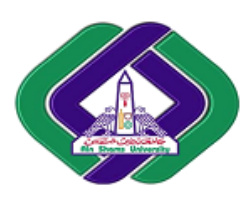

\title{
GLAUCOMA DIAGONSIS FCM_TK ALGORITHM BASED ON FUNDS CAMERA
}

\author{
Mohamed Aouf \\ Ghada Kareem \\ Higher Technological Institute Tenth of Ramadan City, Cairo, Egypt \\ maoufmedical@yahoo.com
}

\begin{abstract}
Glaucoma is considered one of the most important human eye diseases that causes loss of vision. The main objective of this paper is extracting the features from fundus image to evaluate and minimize the risk of Glaucoma. This evaluation based on the Cup-to-Disc Ratio (CDR) of a color retinal fundus image. Actually, CDR is the actual value for the evolution of the risk of Glaucoma. Features used to evaluate the Glaucoma risk are extracted by detection and segmentation process. In this paper a method is proposed for Glaucoma image classification. The proposed method is as follows: the features extracted by discrete wavelet transform (DWT) and then followed by Teager-Kiaser Energy (TKE) operator to improve the detection quality, then classification is done based on fuzzy clustering method. The results showed that the proposed methods succeeded to diagnose the Glaucoma disease with high accuracy compared with the other state of the art techniques.
\end{abstract}

Keywords: - Teager-Kaiser operator energy, Glaucoma, Gabor filter; SVM; K-means, discrete wavelet transform, Clusters.

\section{Introduction}

Image processing is considered one of the most important methods in medical images analysis. Computing analysis and image processing are the most practical and quickly methods which have used features and detection techniques until now. Moreover, Image processing has improved the detection and classifications methods based on some techniques such as the support vector machine, neural network and also discrete wavelet transform. Research has presented the detection of dangerous diseases such as "Glaucoma". Glaucoma is considered one of the most dangerous eye diseases. It is the primary cause of blindness. One of the causes of this disease is the rising of eye pressure and the vision will lose when sustaining this rising pressure for a long time [1].

The most recent research has introduced some of the ideas for detection of the Glaucoma disease to help the doctors in the diagnosis. Some of the researchers have focused on the types of the filter before detection such as in [2]. In [2], authors have presented Gabor transformation as a method for extraction some of the features to help in Glaucoma diagnosis. They have extracted a number of features included mean, variance, skewness, kurtosis, energy, Shannon, Rényi, and Kapoor entropies. The performance of this method has achieved by highest values, an average accuracy of $93.1 \%$, and sensitivity of $89.7 \%$ and specificity of $96.2 \%$ using 23 features with Support Vector Machine (SVM) classifier.

In [3], authors have applied method which focused on optic disk and cup segmentation technique. This method was based on the online database and also, they have updated the system continuously with more clinical groundtruth images.

Moreover, authors in [4] have achieved a novel computational for automatic detection for retinal image analysis and displayed the features of glaucoma and also achieved accurately especially in prediction result. Further, they have proved multi-class categorization training accuracy of $100 \%$ in classifying 45 images. Furthermore, there was a lot of reviews have described the degree of eye diseases and the types of dangerous diseases such as in [5]. 
The authors have applied survey with the percentage of spreading of the eye diseases and they have displayed the types of these diseases.

In some researches, authors have focused on the classification of glaucoma based on the neural networking and they have tried to improve the method to be more accurate such as in [6]. Actually, in [6] authors have tried a new technique for detection based on the artificial neural network. The simulation on MATLAB has approved good accuracy in the detection of glaucoma based on Cup to Disc ratio of retinal fundus images. Further, authors in [7] have achieved a new method for diagnosing glaucoma but only just on suspected glaucoma. The method has proved $93.5 \%$ as the accuracy of detection of suspected glaucoma. Fuzzy logic has considered one of the most technique is very useful in detection and classifications

In [8], authors have applied fuzzy logic method a new expert system for diagnosing glaucoma. They have applied Randomized Hough Transform to extract some of features parameters and also have achieved a classification of these features based on "Fuzzy logic" technique. The accuracy of this method has compared to other methods has achieved more than $94 \%$ especially in prediction. In [9], authors have applied new segmentation technique to extract features of glaucoma called Ocular cup. They achieved high exactness in segmentation process. Furthermore, Teager-Kaiser energy operator has played important role in prediction and feature extraction techniques especially in biomedical system. Some authors as in [10], authors approved that Teager-Kaiser energy operator can be used in detection processing.

In [10], authors have approved the features extraction from ECG signal based on fuzzy cluster based on applying of Teager-Kaiser energy operator. In [11], authors have achieved new method to improve detection based on robust system that which consisted of pre- processing of the signal with Teager-Kaiser operator also, they have approved good accuracy.

In this paper the proposed algorithm is focused on how to extract the features of glaucoma using wavelet transform followed by Teager-Kaiser energy operator. On the other hand, thresholding is used before segmentation. Classification of features based on fuzzy cluster method. The research work of this paper is organized as follows: Sec 2 explains Teager-Kaiser operator energy, the proposed methodology presented in sec3, Results and discussions are presented in sec4

\section{Teager-Kaiser energy operator (TKEO)}

Teager-Kaiser (TK) operator was proposed by Teager to track the energy in speech signals.Teager-Kaiser (TK) operator is a nonlinear quadratic operator on Newton's law of motion used to measure the real physical energy of a system. The total energy was combined, both potential and kinetic. By using The TK energy operator. The energy needed to generate can be estimated, TKEO is used to analyze single component signals from an energy point of view [10], TKEO can be defined in discrete and continuous domain.

The main advantage of this operator is the ability to compute the energy functions of quite complicated function, these functions can be defined as products of the simplest function

The discrete TK energy operator, $\Psi$ is defined in time domain as:

$$
\psi\left(x_{n}\right)=x_{n}^{2}-x_{n+1} x_{n-1}
$$

For an oscillatory signal

$$
x_{n}=A \cos \left(\omega_{n}+\phi\right)
$$

The o/p of TK energy operator given by (li et al.2007) 


$$
\psi\left(x_{n}\right)=A^{2} \sin ^{2}\left(\omega_{n}\right)
$$

The equation above is expressed the energy of an oscillatory signal it deepened on amplitude and frequency values. The nonlinear operator has several features such as: the ability to track instantaneously, varying special patterns, efficiency and simplicity. There are several applications have been derived for one-dimensional and two-dimensional signal processing [11].

\section{Methodology}

The proposed system consists of three main stages: the pre-processing stage, features extraction stage, and the classification process. Fig (1) shows the block diagram of the system each block will be discussed briefly in the following sections.

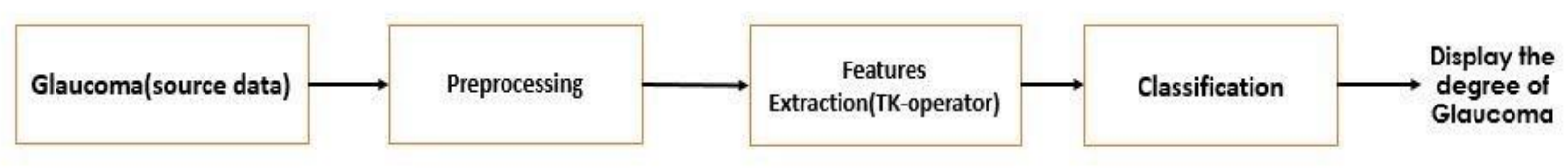

Figure 1: The main block diagram of proposed system

\subsection{Glaucoma database input stage}

The main objective of the proposed system is how to diagnosis the glaucoma, updated the glaucoma using the system continuously with more clinical ground-truth images. The proposed method focuses on optic disk [12]

\subsection{Preprocessing stage}

In this stage, Gabor filter will be applied for denoising the images based on equation (4). The filter has played an important role in this case because the fundus camera in some of the images had some of more brightness and lights so, Gabor filter have tried to enhance the image to be more clearly than before.

$$
g_{\theta}(x)=\frac{1}{\sqrt{2 \pi \sigma}} e^{\frac{-x^{2}}{2 \sigma^{2}} \cos \left(2 \pi w_{0} x\right)}
$$

The pre-processing stage has included the segmentation process and actually, the segmentation process has achieved by the thresholding method

Otsu thresholding method have approved as the best method for image segmentation ${ }^{[13] .}$

\subsection{Feature extraction stage}

This process has divided into two stages: First stage is how to extract features based on discrete wavelet transform. Second stage is how to improve this stage of feature extraction using Teager-Kaiser operator energy (TK).

Actually, discrete wavelet transform has played an important role in the extraction process. DWT is applied on the pre-processed image based on equation (5) [14].

$$
\operatorname{DWT}(\mathrm{m}, \mathrm{k})=\frac{1}{a} \sum_{m=0}^{N=1} s(n) g\left(\frac{k-b}{a}\right)
$$


Where $s(n)$ is the binary segmented image, $(a, b)$ are the scaling parameters, $m$ is the decomposition level, $g(\cdot)$ is the mother wavelet function and $\mathrm{N}$ is the number of features.

The operator Teager-Kaiser has been used in classification using fuzzy cluster method and also in prediction technique $[10,15]$.

\subsection{Classification stage}

In this stage, the degree of glaucoma is classified based on fuzzy clustering method [14]. The fuzzy cluster method is applied and approved. The equation of fuzzy cluster is as shown in equation (7) [16-21].

The main operation of classification is applied based on the following steps:

First step is to determine the clusters after partitioning the data into fuzzy groups where the vector of data $\mathrm{x}_{\mathrm{i}}=1$, $2, \ldots \mathrm{n}$, then, the second step is determining the center of each cluster $\mathrm{c}_{\mathrm{i}}=1,2, \ldots \mathrm{c}$, and then, determining the membership function from the following equation (6) :

$$
\mu_{i j}=\frac{1}{\sum_{k=1}^{c}\left({ }^{d_{i j}} / d_{k j}\right)^{2 / m-1}}
$$

After that, we have to determine the objective function from:

$$
\sum_{i=1}^{c} J_{i}=\sum_{i=1}^{c} \cdot \sum_{i=1}^{c} \mu_{i j}^{m} d_{i j}^{2}
$$

Where $i=[1,2, . ., c]$ is the number of clusters selected from data points $n, j$ is the number of objects $m^{E}[1, \infty]$, $\mu_{i j}$ is the member function for object $\mathrm{j}$ and cluster $\mathrm{i}$, and $\mathrm{d}_{\mathrm{ij}}=\left\|c_{i}-x_{j}\right\|$ is the Euclidean distance between $\mathrm{c}_{\mathrm{i}}$ and $\mathrm{x}_{\mathrm{j}}$.

Finally, Compute the new c-fuzzy cluster using the following equation (7) [18]:

$$
c_{i}=\frac{\sum_{j=1}^{c} \mu_{i j}^{m} x_{j}}{\sum_{j=1}^{c} \mu_{i j}^{m}}
$$

\subsection{The main algorithm:}

The block diagram, given in Figure (1), is programmed using Python. In the following, the general algorithm of the system is described by the flow chat as in Figure (3). Figure 2 is the pseudo code for the proposed algorithm.

\section{Start}

Apply PIL (Python Library ) to import our database test or images of fundus camera. Apply Gray scale level for colored images. Create

Thumbnails (from PIL ).

Convert to grayscale level. 
IJICIS, Vol.18, No. 2

Applying Gabor filter ( a Gaussian kernel) and we have used Matplotlib . Extract the

segmentation in separate image.

Applying DWT functions and apply TK- our structure code

Applying FCM (Fuzzy logic functions based on C-means ) End

Figure 2: pseudo code for the proposed algorithm

The cup-to- Disc ratio (CDR) is measured to estimate the progression of glaucoma, the optic disc may have an amount of cupping or flat. Glaucoma is affected on CDR where it produced pathological cupping of the optic disc $[22,23]$, when glaucoma increased, the cup maximized till it full the disc area the normal value of cup- to disc ratio is 0.3 if it is increased, The patient will suffer from the glaucoma . 


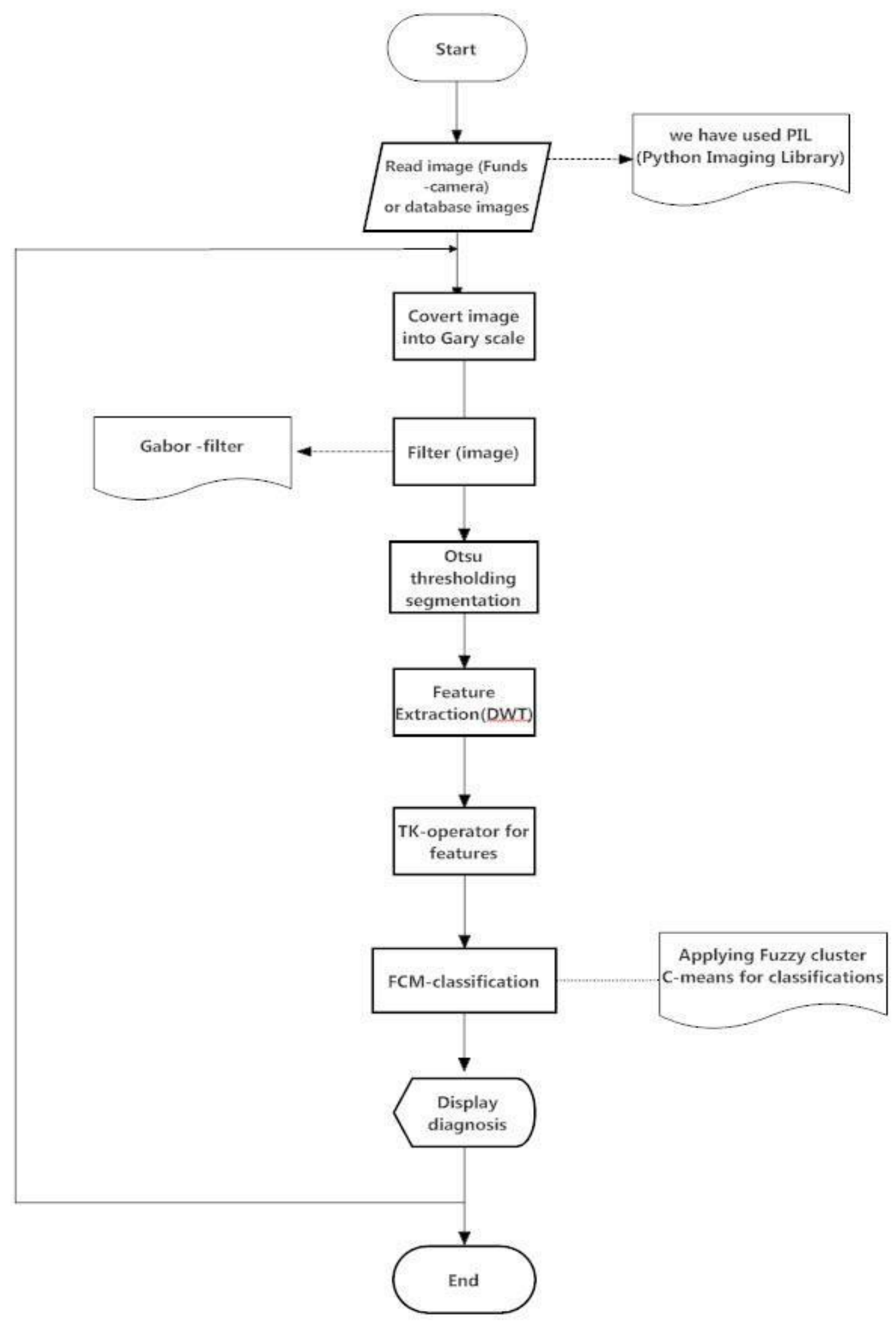

Figure 3: The Flow chart of system 


\section{Results and Discussion}

The proposed methodology is based on two categories the first was based on -online database, and the other was based on fundus camera images. From pervious sections, Gabor filter method was applied to filter images glaucoma especially from fundus camera. The segmentation processes were applied based on thresholding technique and in this approach a set of calculations is done. The figures from figure (4) to figure (6) shows the process of segmentation before the feature extraction method. Figure (3) indicates the image before gray level, in figure (6) after calculations the optic disc is displayed and the diameter of optic disc can be determined. Increasing of diameter of optic disc is indicated that the patient suffers from glaucoma actually, in the feature extraction method DWT with TK- operator was applied to improve the extraction technique as the pervious researches in the other applications. Furthermore, we have compared our work after classification with the others work and the result showed that the proposed technique achieved more than 95\% classification accuracy using TK- operator with fuzzy cluster operation method.

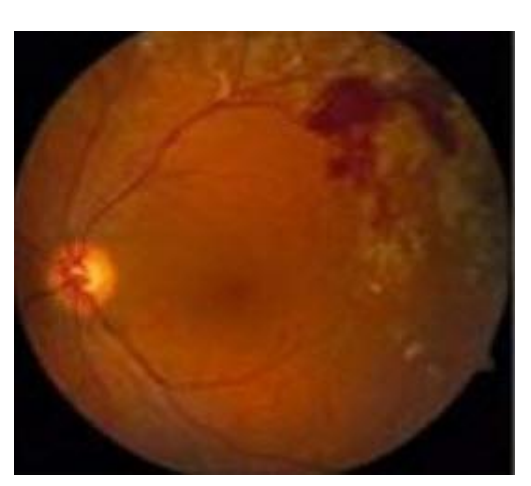

Figure 4: Image before gray level

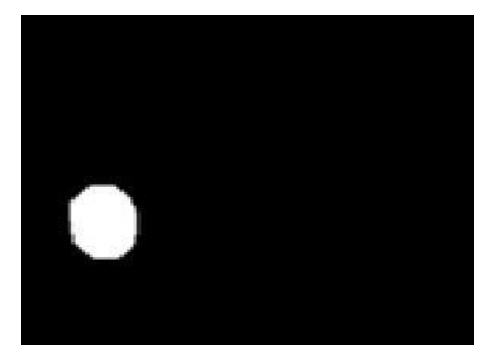

Figure 6: Image after Threshoding and and display Morphological operation

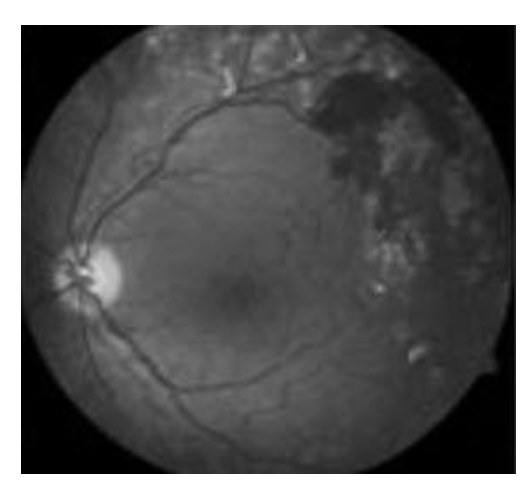

Figure 5: Image in gray scale

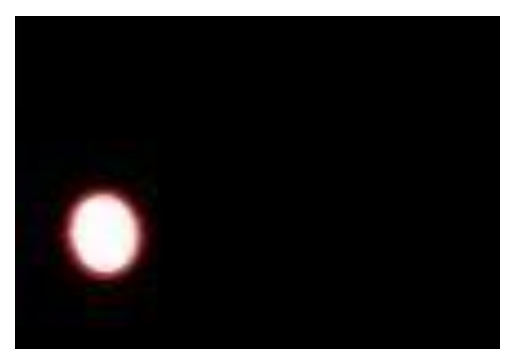

Figure 7: Image after calculation the optic disk diameter

\begin{tabular}{|c|c|c|c|}
\hline \multicolumn{2}{|c|}{ Proposed method } & $\begin{array}{c}\text { Glaucoma } \\
\text { detection using } \\
\text { cup to disc ratio } \\
{[6]}\end{array}$ & $\begin{array}{c}\text { image processing } \\
\text { algorithms for early } \\
\text { glaucoma } \\
\text { diagnosis }^{[8]}\end{array}$ \\
\hline accuracy & $95 \%$ & $93.5 \%$ & $94 \%$ \\
\hline CDR & 0.29 & 0.34 & - \\
\hline
\end{tabular}


In this paper diagnosis of glaucoma is based on the CDR is done, the system was tested on dataset of 120 images and the accuracy is high when compared to others, i.e. Proposed methods succeeded to detect the Glaucoma disease with high accuracy.

The accuracy can be defined as the percentage of correctly classified instances $(\mathrm{TP}+\mathrm{TN}) /(\mathrm{TP}+\mathrm{TN}+\mathrm{FP}+\mathrm{FN})$. where $\mathrm{TP}, \mathrm{FN}, \mathrm{FP}$ and TN represent the number of true positives, false negatives, false positives and true negatives, respectively

Confusion Matrix

\begin{tabular}{|c|c|c|c|}
\hline & \multicolumn{2}{|c|}{ Predicted } \\
\hline & & ROI & Background \\
\hline Ground & ROI & TP & FN \\
\hline Truth & Background & FP & TN \\
\hline
\end{tabular}

Accuracy $=(\mathrm{TP}+\mathrm{TN}) /(\mathrm{TP}+\mathrm{FN}+\mathrm{FP}+\mathrm{TN})$

Over segmentation $=\mathrm{FP} /(\mathrm{TP}+\mathrm{FP}+\mathrm{FN})$

Under segmentation $=\mathrm{FN} /(\mathrm{TP}+\mathrm{FP}+\mathrm{FN})$

\begin{tabular}{|l|l|r|r|}
\cline { 3 - 4 } \multicolumn{2}{c|}{} & \multicolumn{2}{c|}{ Predicted } \\
\cline { 3 - 4 } \multicolumn{2}{c|}{} & $\begin{array}{l}\text { Optical } \\
\text { Disc }\end{array}$ & Background \\
\hline $\begin{array}{l}\text { Manual } \\
\text { Segmentation }\end{array}$ & Optical & 2019 & 1102 \\
& Disc & & 80988 \\
\cline { 2 - 4 } & Background & 3507 & \\
\hline
\end{tabular}

Accuracy $=(2019+80988) /(2019+1102+3507+80988)=83007 / 87616=0.94739$

Over segmentation $=3507 /(2019+1102+3507)=0.529$

Under segmentation $=1102 /(2019+1102+3507)=0.16626$

\section{Conclusion and future work}

In the proposed system, algorithm is implemented to improve the diagnosis process of glaucoma by extracting the features using DWT which followed by Teager-Kaiser operator energy and that was helped in classification technique and the system is achieved a good accuracy when fuzzy cluster method is applied. The proposed system can be used with doctor, it can minimize the human error and diagnosis the glaucoma. In future we can enhancement the accuracy, and apply the system to diabetic person

\section{Data Availability}

Our work in this paper have based on two sources of data as described before one of them is an online clinical database such as in [12] and the other is fundus camera images.

\section{References}




\section{IJICIS, Vol.18, No. 2}

[1] Schuman, J. S., Hee, M. R., Arya, A. V., Pedut-Kloizman, T., Puliafito, C. A., Fujimoto, J. G., \& Swanson, E. A. Optical coherence tomography: a new tool for glaucoma diagnosis. Current opinion in ophthalmology, 6(2), 89-95, 1995.

[2] Acharya, U. R., Ng, E. Y. K., Eugene, L. W. J., Noronha, K. P., Min, L. C., Nayak, K. P., \& Bhandary, S. V. Decision support system for the glaucoma using Gabor transformation. Biomedical Signal Processing and Control, 15, 18$26,2015$.

[3] Zhang, Z., Yin, F. S., Liu, J., Wong, W. K., Tan, N. M., Lee, B. H., \& Wong, T. Y.Origa-light: An online retinal fundus image database for glaucoma analysis and research. InEngineering in Medicine and Biology Society (EMBC), Annual Inter-national Conference of the IEEE (pp. 3065-3068). 2010

[4] Ramani, R. G., Balasubramanian, L., \& Jacob, S. G. Automatic predic-tion of Diabetic Retinopathy and Glaucoma through retinal image analysis and data mining techniques. In Machine Vision and Image Processing (MVIP), 2012 International Conference on (pp. 149-152). IEEE, 2012.

[5] Ravudu, M., Jain, V., \& Kunda, M. M. R. Review of image processing techniques for automatic detection of eye diseases. In Sensing Technology (ICST), 2012 Sixth International Conference on (pp. 320-325). IEEE, 2012.

[6] Gayathri, R., and P. V. Rao. "Glaucoma detection using cup to disc ratio and artificial neural networks." International Journal of Engineering \& Technology 7.1.5: 135-140, 2017.

[7] Sengar, N., Dutta, M. K., Burget, R., \& Ranjoha, M. Automated detection of suspected glaucoma in digital fundus images. In Telecommunications and Signal Processing (TSP), July 2017 40th International Conference on (pp. 749-752). IEEE, 2017.

[8] Soltani, A., Battikh, T., Jabri, I., \& Lakhoua, N. A new expert system based on fuzzy logic and image processing algorithms for early glaucoma diagnosis. Biomedical Signal Processing and Control,40, 366-377,2018.

[9] Bokhari, F., Syedia, T., Sharif, M., Yasmin, M., \& Fernandes, S. L. Fundus Image Segmentation and Feature Extraction for the Detection of Glaucoma: A New Approach. Current Medical Imaging Reviews, 14(1), 77-87, 2018.

[10] Nady, S., Moness, M., Massoud, M., \& Gharieb, R. R. Combining continuous wavelet transform and TeagerKaiser Energy operator for ECG arrhythmia detection. In Biomedical Engineering Conference (CIBEC), 2016 8th Cairo International (pp. 76-79). IEEE, 2016.

[11] Manresa, José A. Biurrun, Carsten D. Mørch, and Ole K. Andersen. Teager-kaiser energy operator improves the detection and quantification of nociceptive withdrawal reflexes from surface electromyography. Signal Processing Conference, 2010 18th European. IEEE, 2010.

[12] http://www5.cs.fau.de/research/data/fundus-images

[13] Cho, C. W., Lee, J. W., Shin, K. Y., Lee, E. C., Park, K. R., Lee, H., \& Cha, J. Gaze Detection by Wearable EyeTracking and NIRLED-Based Head-Tracking Device Based on SVR'. ETRI Journal, 34(4), 542-552, 2012. 
[14] Rahouma, K. H., Muhammad, R. H., Hamed, H. F., \& Eldahab, M. A. A. Analysis of electrocardiogram for heart Performance Diagnosis Based on Wavelet Transform and Prediction of Future Complications. Egyptian Computer Science Journal (ISSN-1110-2586), 41(2), 2017.

[15] Rankawat, S. A., Rankawat, M., \& Dubey, R. ECG artifacts detection in non- cardiovascular signals using Slope Sum Function and Teager Kaiser Energy. In BioSignal Analysis, Processing and Systems (ICBAPS), 2015 International Conference on (pp. 6-10). IEEE, 2015.

[16] De Sarkar, S. C., Chattopadhyay, S., \& Pratihar, D. K. A comparative study of fuzzy c-means algorithm and entropy-based fuzzy clustering algorithms, 2011.

[17] $\mathrm{Au}, \mathrm{W} . \mathrm{H}, \mathrm{A}$ Distributed Algorithm for Mining Fuzzy Association Rules in Traditional Databases. In Handbook of Research on Fuzzy Information Processing in Databases (pp.685-705). IGI Global, 2008.

[18] De Sarkar, S. C., Chattopadhyay, S., \& Pratihar, D. K. Comparative study of fuzzy c-means algorithm and entropy-based fuzzy clustering algorithms, 2011.

[19] Dallali, A., Kachouri, A., \& Samet, M. (2011). Fuzzy c-means clustering, Neural Network, wt, and Hrv for classification of cardiac arrhythmia. ARPN Journal of Engineering and Applied Sciences, 6(10), 2011.

[20] Hsieh, C. C., Hsih, M. H., Jiang, M. K., Cheng, Y. M., \& Liang, E. H. (2016). Effective semantic features for facial expressions recognition using SVM. Multimedia Tools and Applications, 75(11), 6663-6682, 2016.

[21] Hu, W., Huang, Y., Wei, L., Zhang, F., \& Li, H. Deep convolutional neural net-works for hyper spectral image classification , 2015

[22] Glaucoma causes Optic Nerve Cupping (atrophy) and Vision Loss. The Eye Digest. The university of Illinois Eye and Ear Infirmary Archived from the original on 8 July 2012

[23] Eye Exam and tests for Glaucoma diagnosis". The Eye Digest. The University of Illinois Eye and Ear Infirmary. Archived from the original on 8 July 2012. 